\title{
Comet-tailed drusen of the retinal pigment epithelium in angioid streaks
}

\author{
Purpose To describe a fundus finding in \\ angioid streaks, namely comet-tailed drusen of \\ the retinal pigment epithelium. \\ Methods A review was carried out of fundus \\ photographs in $\mathbf{1 1 0}$ cases of angioid streaks. \\ Results Five patients had comet-tailed drusen \\ and the halo tail was radial and parallel to \\ adjacent angioid streaks. \\ Conclusions Comet-tailed drusen may \\ represent zones of minor stress of the retinal \\ pigment epithelium-Bruch's membrane \\ complex compared with zones of major stress \\ as in angioid streaks.
}

Key words Angioid streaks, Bruch's membrane, Drusen of the retinal pigment epithelium

\section{Angioid streaks have a characteristic} appearance of linear, narrow subretinal streaks radiating a few millimetres from a peripapillary ring. There is a high incidence of macular degeneration at a young age in eyes with angioid streaks. Several retinal findings are associated with angioid streaks, such as peau $\mathrm{d}^{\prime}$ orange appearance ${ }^{1-3}$ and large optic disc drusen. ${ }^{4,5}$ To that list, we would like to add comet-tailed drusen of the retinal pigment epithelium. ${ }^{6}$

In a retrospective manner, we reviewed the fundus photographs of 110 consecutive patients from 110 families with angioid streaks (58 women and 52 men; 95 whites and 15 blacks; mean age 50 years, range $8-77$ years; 26 patients had pseudoxanthoma elasticum). Fundus findings included peau d'orange appearance $(55.5 \%)$, peripapillary atrophy $(26.4 \%)$, pigment proliferation along the angioid streaks (14.5\%), peripheral retinal scars $(11.8 \%)$ and optic nerve head drusen $(6.4 \%)$. Twenty patients had retinal pigment epithelial drusen in the macula or the periphery (some from age-related macular degeneration), among which 10 had calcified drusen in and around the angioid streaks. These 10 patients were not different from the 100 remaining patients as they comprised: 6 men and 4 women, 8 whites and 2 blacks, mean age 39 years and range 18-62 years, 3 patients with pseudoxanthoma elasticum, and 7 patients with the peau d'orange appearance. The drusen were associated in these 10 patients with the presence of helicoid peripapillary atrophy (2 cases), diffuse angioid streaks or eggshell appearance (3 cases), and very wide angioid streaks (2 cases). Five of these 10 patients had drusen of the retinal pigment epithelium (50-200 $\mu \mathrm{m}$ in diameter) with a centripetal (from the drusen towards the optic disc) comet-shaped halo (retinal pigment epithelial atrophy 50-200 $\mu \mathrm{m}$ in width). The halo was radial and parallel to adjacent angioid streaks (Fig. 1). The finding was multiple (5 lesions dispersed on either side of a very wide angioid streak) in one patient only, and singular in the rest.

Drusen of the retinal pigment epithelium occur in about 1 of 7 eyes with angioid streaks. ${ }^{1}$ Atrophy of the retinal pigment epithelium around drusen of the retinal pigment epithelium occurs in age-related macular degeneration. Atrophy of the retinal pigment epithelium occurs around angioid streaks in $10 \%$ of subjects in the form of helicoid peripapillary atrophy. ${ }^{1}$ Histopathologically, angioid streaks correspond to a full-thickness break in Bruch's membrane with disruption of the underlying choriocapillaris and atrophy of the overlying retinal pigment epithelium. Bruch's membrane in angioid streaks is thickened and basophilic from deposition of calcium. ${ }^{7,8}$ The deposition of calcium confers an eggshell brittleness to Bruch's membrane that is modulated by the axis of the optic nerve insertion, resulting in radiating cracks on Bruch's membrane. ${ }^{9}$ Comet-tailed drusen appear more commonly in eyes with angioid peripapillary atrophy ('long-standing' angioid streaks). It is hypothesised that the comet-tailed centripetal halo represents a line of stress below the threshold needed to cause visible cracks in Bruch's membrane. Indocyanine green angiography is helpful in detecting abnormalities in the retinal pigment epithelium basement membrane. ${ }^{10}$ An indocyanine green angiographic study of comet-tailed halos may add more information to their pathogenesis and probably would demonstrate a small rupture of Bruch's membrane under the halos.
A.M. Mansour, M.D. Department of Ophthalmology American University of Beirut Beirut, Lebanon

W.H. Annesley Wills Eye Hospital Philadelphia USA 


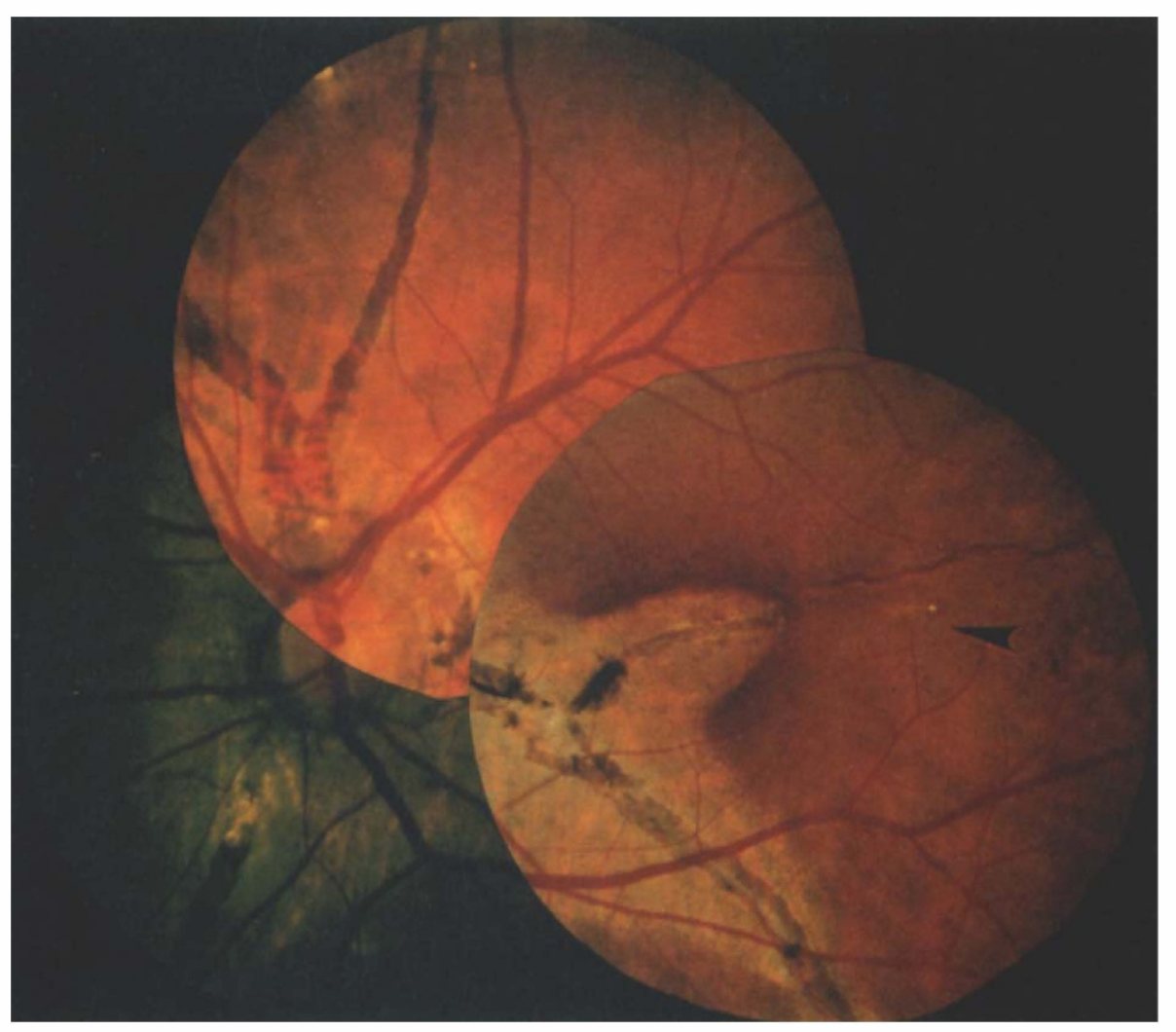

Fig. 1. Drusen of the retinal pigment epithelium with a comet-like halo continuous with an angioid streak.

\section{References}

1. Mansour AM, Ansari NH, Shields JA, et al. Evolution of angioid streaks. Ophthalmologica 1993;207:57-61.

2. Krill AE, Klien BA, Archer DB. Precursors of angioid streaks. Am J Ophthalmol 1973;76:875-9.

3. Shimizu K. Mottled fundus in association with pseudoxanthoma elasticum. Jpn J Ophthalmol 1961;5:1-13.

4. Mansour AM. Is there an association between optic disc and angioid streaks? Graefes Arch Clin Exp Ophthalmol 1992;230:595-6.

5. Coleman K, Ross MH, McCabe M, et al. Disk drusen and angioid streaks in pseudoxanthoma elasticum. Am J Ophthalmol 1991;112:166-70.
6. Gass JDM. Stereoscopic atlas of macular diseases. St Louis: CV Mosby, 1987:102-12.

7. Jampol LM, Acheson R, Eagle RC, et al. Calcification of Bruch's membrane in angioid streaks with homozygous sickle cell disease. Arch Ophthalmol 1987;105:93-8.

8. Dreyer, R, Green WR. The pathology of angioid streaks: a study of 21 cases. Trans Pa Acad Ophthalmol Otolaryngol 1978;31:158-67.

9. Mansour AM. Systemic associations of angioid streaks. Int Ophthalmol Clin 1991;31:61-8.

10. Kim DD, Pulido JS, Wipplinger WA. Indocyanine green angiographic findings in pseudoxanthoma elasticum. Am J Ophthalmol 1993;116:767-9. 